PSICOLOGIA Vol. IX $\mathrm{N}^{2} 11991$

\title{
ORIENTACION DEL ROL SEXUAL EN MUJERES QUE TRABAJAN EN PROFESIONES SEXUALMENTE TIPIFICADAS
}

Miriam Baruch Goldstein*

Se compararon 288 mujeres peruanas de profesiones tradicionalmente masculinas y femeninas, en la autopercepción de su masculinidad y femineidad a nivel global y en los contextos familiar y laboral. Se empleó la Escala de Orientación Sexual de Ragúz, SROS (1989) y versiones modificadas de la misma. Si bien el análisis de varianza y el t Student no evidenciaron diferencias en la ORS global o familiar, los grupos difirieron en su ORS laboral.
Two hundred eighty-eight Peruvian women from traditionally masculine and feminine professions were compared on their global, family and work sex role orientation (Raguz' SROS, 1989). Analysis of variance and t-tests showed significant differences in sex role orientation only in the work context. Results are discussed. 

Las nuevas alternativas de trabajo que se ofrecen para la mujer plantean una reconsideración de los conceptos tradicionales acerca de los roles sexuales. En nuestra sociedad se tiende a considerar que las mujeres deben ser cálidas, agradables, sensitivas, emocionales y sumisas frente a los hombres. Ellos, por el contrario, deben ser competentes, no emocionales, lógicos, dominantes, independientes y agresivos (Bonino, Kiguel, Santelices, 1980).

Son estos estereotipos percibidos los que nos llevan a considerar el nuevo mundo profesional de la mujer, al que cada vez va teniendo mayor acceso. ¿Qué pasa con aquellas mujeres que incursionan en "otros", ambientes como la ingeniería, la medicina, la administración? ¿Cómo se autoperciben en comparación a los modelos tradicionales?.

El objetivo de nuestro trabajo fue el estudio de la autopercepción de la mujer respecto de su masculinidad y femineidad tanto en la esfera familiar como laboral. Nuestra pregunta central fue saber si existían diferencias entre la orientación de rol sexual (ORS) a nivel global y la ORS en los contextos familiar y laboral entre mujeres que trabajan en profesiones tradicionalmente femeninas y tradicionalmente masculinas.

El concepto de identidad de rol sexual es un tanto complejo ya que, dependiendo del marco conceptual con el que se trabaje, puede aludir a varios niveles de comprensión o funcionamiento entrelazados: la orientación del rol, que es la autopercepción de la propia masculinidad y femineidad; la preferencia que se da a nivel del deseo o motivación determinada; y la adopción que es más bien a un nivel conductual, refiriéndose a la postura que el individuo asume y es percibida por los demás (Schaffer, 1979). Los instrumentos de medición no siempre evidencian las distinciones necesarias (Spence y Helmreich, 1979). Best (1980), Williams (1981) y Williams y Best (1982) revelan estudios en Lima con muestras específicas de universitarios y niños de clase media-alta y alta, a partir de las cuales concluyen que el Perú tiene una estereotipia sexual intermedia en relación a otros 25 países. Sin embargo, la situación en el país presenta grandes diferencias a nivel regional (Campaña, 1982). 
En cuanto a la situación de la mujer que trabaja, Sara-Lafosse (1978) describe la existencia de un marco social capitalista y dependiente donde prevalecen estilos de relaciones patriarcales.

Con la difusión de las ideas feministas y el avance en la investigación se empezaron a considerar las variables de género y "división sexual del trabajo" constatándose entonces la "existencia de mercados laborales segmentados por sexo" (Barrig, 1986, p. 3).

Scott (en Barrig, 1988) realizó una de las primeras investigaciones sobre el empleo femenino desde la mira de la división del trabajo por género. Para la autora, la participación laboral no estaría delimitada por la perspectiva de la modemización (educación y capacitación) ni la teoría de la dependencia, sino por el crecimiento de la demanda de "trabajo de mujeres". Las ocupaciones que involucran poder, prestigio, iniciativa y responsabilidad no son ocupadas por ellas, manteniéndose esta situación pese a una mayor escolarización en el período 1961-1972. El desarrollo económico benefició más a las mujeres de sectores medios, requiriéndose de profesoras, enfermeras y trabajadoras sociales. Pese a una nueva visión económica y educativa, los trabajos estereotipados para cada sexo se mantuvieron, así como la segregación de mercados laborales por género.

El "boom" de los años "60 llevó a la mujer a incursionar en distintos tipos de trabajos. Era de esperar que éstas transformaciones afectaran las costumbres y normas en pos de una mayor aceptación del trabajo de la mujer fuera de casa. Sin embargo, tal cambio no se dio. La estricta concepción sobre una división sexual del trabajo persistió permaneciendo la gran mayoría de las mujeres en trabajos a nivel doméstico, y reproduciendo posteriormente en otras esferas la segregación y subordinación (Guzmán, 1978)

Sin embargo, es importante señalar que una revisión de trabajos realizados en el Perú durante los últimos 15 años sobre género e identidad femenina, desde la perspectiva psicológica y antropológica, describe una situación de cambio donde parecería que la mujer está afirmando nuevos rasgos de identidad (Lora y Anderson, 1989).

Sin perder de vista la dimensión de la profesionalización de la mujer y el logro de una identidad ocupacional, es importante tener en cuenta que las elecciones vocacionales involucran variables tales como valores, identificaciones, necesidades y capacidades, que engloban a toda la personalidad. La identidad ocupacional también puede ser comprendida en términos de roles desingnándose a las ocupaciones como el conjunto de expectativas que tiene la sociedad sobre el rol de un individuo (Bohoslavsky, 1974). En la elección 
de dichas ocupaciones también se ve en juego la estereotipia de identidad sexual "ya que hay ocupaciones más o menos masculinas o femeninas y el adolescente integra esta valoración a su identidad ocupacional" (op. cit., p. 50).

Algunos factores que pueden estar interviniendo en la vocación profesional de muchas mujeres son las limitaciones a sus aspiraciones por la dificultad posterior para hallar empleo, el carácter discriminatorio de la educación y la insuficiente información sobre las nuevas oportunidades de trabajo (Unicef, 1981). Como factores subjetivos se señalan el hecho de que muchas mujeres no se dedican a carreras de largos años de formación porque prevalece el interés de búsqueda de pareja para constituir un matrimonio, así como la preferencia por ocupaciones donde se puede alternar socialmente. Además se tiende a escoger el mínimo de esfuerzo físico y condiciones de trabajo agradables.

Desde la perspectiva de los roles sexuales son relevantes los aportes referidos a la elección vocacional. En un estudio con universitarios canadienses cuyas madres poseen una visión tradicional, se encuentra un predominio de metas ocupacionales modestas (Sutherland, 1978). La dicotomía entre la vida afectiva y la realización profesional es estudiada en una investigación Iraní donde las mujeres muestran una mayor preocupación por el matrimonio y relaciones con el sexo opuesto que por la vida profesional, acentuándose esta situación en los niveles de mayor discriminación de la mujer y menor nivel socioeconómico (Tohidi, 1984) Parece ser, entonces, que el grado de tradicionalismo es afín al del nivel educativo, en cuanto a preferencias e intereses (Hawley \& Even, 1982).

Sin embargo, no en todos los casos de madres profesionales los hijos las consideran modelos ocupacionales. En un estudio con abogadas graduadas entre 1920 y 1979 se observó que solamente cuando el cónyuge también era profesional , los hijos tendían a seguir el modelo familiar (Dambrot \& Vassel, 1983).

En cuanto a las elecciones vocacionales según rasgos de personalidad, se ha encontrado que las mujeres que siguen carreras de Ciencias Sociales tienen una orientación de rol más femenina, más intereses sobre la familia y mayor flexibilidad para el trabajo. Aquellas que siguen Ingeniería, tienen mayores incentivos pero también es mayor su necesidad de tener hijos y ser buenas madres (Galejs \& King, 1983)

En base a los conceptos esbozados y las investigaciones mencionadas, nos preguntamos si la Orientación de Rol Sexual (ORS) de mujeres de profesiones tradicionalmente femeninas es diferente de las mujeres de profesiones masculinas. Más aún, nos interesó explorar las posibles diferencias en la ORS en lns contextos familiar y laboral. 


\section{Metodología}

Los sujetos fueron 288 mujeres profesionales entre 20 y 40 años que ejercen en seis profesiones femeninas y masculinas, -en terminos tanto de distribución estadística como de estereotipia de las profesionales mismas-. Se controlaron las variables de estado civil (soltera/casada), maternidad y edad de los hijos (sin hijos/hijos preescolares/hijos escolares). La mayoría de las mujeres resultó tener entre uno y cinco años de graduadas, y muy pocas tenían más de diez.

Se utilizaron lo siguientes instrumentos:

1. Ficha Demográfica: edad, ocupación actual, estado civil, número de hijos e historia.

2. Escala de Orientación del Rol Sexual de Ragúz SROS (Ragúz et al., 1989): instrumento de autoreporte tipo Likert, derivado del Inventario de Roles Sexuales de Bem (BSRI), compuesto por 53 reactivos correspondientes a:

- una escala de orientación de rol sexual, con 18 ítem, (SROS-F)

- dos subescalas de orientación de rol sexual masculina, una de nueve ítem, (SROS-M1) y otra de ocho item, (SROS-M2).

- una escala neutra de 18 ítem, (SROS-N).

3. SROS Contextual: Se modificó la consigna del SROS para evaluar la ORS:

- En la esfera familiar- doméstica: SROS (F) "En el hogar me considero..."

- En la esfera laboral: SROS (L) "En el trabajo me considero..."

4. Escalas de valoración de los roles y satisfacción personal, construídas por la autora.

El procedimiento consistio en la aplicación de los instrumentos en una sola sesión de manera grupal o individual, según las posibilidades de obtención de la muestra en los distintos centros de trabajo. Cada mujer profesional respondio a una de las formas del instrumento (SROS-G, F, o L).

\section{Resultados}

Para la validación de las hipotesis de la investigación se calcularon las pruebas $\mathbf{t}$ de Student comparando las orientaciones de rol sexual femenino y masculino (en su dos tipos) en las mujeres de profesiones masculinas y feme- 
ninas. No se evidenciaron variaciones en la ORS global (Cuadro 1), ya que no existen diferencias estadísticamente significativas ni en la femeneidad ni en la masculinidad; por lo tanto, no podemos postular que la ORS varía según el tipo de ocupacion sexualmente tipificada.

\section{Cuadro 1}

Pruebas $\mathrm{t}$ de Student de las Escalas del SROS General entre las profesiones masculinas y femeninas

\begin{tabular}{|c|c|c|c|c|c|}
\hline \multirow[b]{2}{*}{ ORS - G } & \multicolumn{2}{|c|}{ Prof. Femenina* } & \multicolumn{2}{|c|}{ Prof. Masculina } & \\
\hline & $\bar{X}$ & DS & $\overline{\mathrm{X}}$ & DS & \\
\hline Femenina & 88.08 & 9.14 & 85.91 & 9.43 & $\begin{array}{l}\mathrm{t}=1.392 \\
\mathrm{~g} 1=94 \\
\mathrm{p}=.082\end{array}$ \\
\hline Masculina 1 & 46.77 & 7.63 & 47.54 & 7.80 & $\begin{array}{l}\mathrm{t}=0.484 \\
\mathrm{~g} 1=94 \\
\mathrm{p}=.317\end{array}$ \\
\hline Masculina 2 & 85.38 & 6.78 & 36.63 & 6.66 & $\begin{array}{l}\mathrm{t}=0.902 \\
\mathrm{~g} \mathrm{l}=94 \\
\mathrm{p}=.314\end{array}$ \\
\hline
\end{tabular}

* 144 mujeres de profesiones "femeninas" y 144 de "masculinas".

Tampoco podemos apreciar diferencias en la femineidad o masculinidad en el contexto familiar (Cuadro 2), de modo que no es posible afirmar la variación de la ORS en el contexto familiar según el tipo de ocupación sexualmente tipificada.

\section{Cuadro 2}

Pruebas $t$ de Student de las Escalas del SROS Familiar entre las profesiones masculinas y femeninas

\begin{tabular}{l|lr|rr|l}
\hline \multirow{2}{*}{ ORS - F } & \multicolumn{2}{|c|}{ Prof. Femenina } & \multicolumn{2}{|c|}{ Prof. Masculina } & \\
\cline { 2 - 5 } Femenina & $\bar{X}$ & DS & $\bar{X}$ & DS & \\
\hline Masculina 1 & 46.85 & 11.97 & 86.65 & 10.26 & $\begin{array}{l}\mathrm{g} 1=94 \\
\mathrm{p}=.282\end{array}$ \\
\hline Masculina 2 & 34.90 & 8.06 & 46.92 & 7.11 & $\begin{array}{l}\mathrm{t}=0.040 \\
\mathrm{~g} 1=94 \\
\mathrm{p}=.484\end{array}$ \\
\hline
\end{tabular}


En el cuadro 3 se analiza la variación de la ORS en el contexto laboral en las ocupaciones estudiadas. Sólo en el caso de la mujeres que trabajan en ocupaciones tradicionalmente masculinas, encontramos una diferencia estadísticamente significativa al analizar la masculinidad 2, (p. 01).

\section{Cuadro 3}

Pruebas $\mathrm{t}$ de Student de las Escalas del SROS Laboral entre las profesiones masculinas y femeninas

\begin{tabular}{|c|c|c|c|c|c|}
\hline \multirow[b]{2}{*}{ ORS - L } & \multicolumn{2}{|c|}{ Prof. Femenina } & \multicolumn{2}{|c|}{ Prof. Masculina } & \\
\hline & $\bar{X}$ & DS & $\bar{X}$ & DS & \\
\hline Femenina & 86.56 & 9.82 & 85.50 & 8.72 & $\begin{array}{l}\mathrm{t}=0.555 \\
\mathrm{~g} \mathbf{1}=94 \\
\mathrm{p}=.294\end{array}$ \\
\hline Masculina 1 & 47.40 & 7.39 & 48.75 & 6.40 & $\begin{array}{l}\mathrm{t}=0.950 \\
\mathrm{~g} 1=94 \\
\mathrm{p}=.327\end{array}$ \\
\hline Masculina 2 & 35.23 & 8.00 & 38.56 & 5.47 & $\begin{array}{l}\mathrm{t}=2.358 \\
\mathrm{~g} 1=94 \\
\mathrm{p}=.010\end{array}$ \\
\hline
\end{tabular}

Por otro lado, el análisis de las escalas adicionales mediante un análisis de varianza simple revela de qué manera dichas escalas se diferencian entre cada profesión estudiada. Vemos entonces que la mayoría de profesionales tanto de profesiones tradicionalmente femeninas como masculinas, tenían madres no profesionales, y las madres profesionales tenían ocupaciones tradicionales.

Se observa también que aunque casi un tercio cree que su madre fue indiferente ante la elección de su profesión, un buen porcentaje (45\%) refiere que ambos padres estuvieron conformes con la elección. Más de la mitad (58\%) considera que los amigos valoran su ocupación (inclusive la valoran mucho, en una cuarta parte de los casos).

La estereotipia ocupacional no influyó en la satisfacción laboral, ya que la mayor y menor satisfacción se encontró en profesiones femeninas (educación y enfermería, respectivamente). Justamente fueron las educadoras - junto con lo médicos- los que mayor valoración del rol laboral percibieron de parte del esposo; y las enfermeras, las que menos valoradas se sienten. El rol de ama de casa reflejó cierto grado de satisfacción, sin que variaran los grupos profesionales. 
En cuanto a las influencias parentales, no se observaron diferencias a nivel de profesiones tradicionales o no, pero sí a nivel específico. La influencia de la madre sobre su ORS es percibida como mayor en las enfermeras, y menor en médicos y obstétrices. El padre no ejerce una influencia diferencial.

Todas las profesionales se sintieron igualmente libres para escoger su profesión.

\section{Discusión}

Hemos visto que los resultados que en términos generales no existen diferencias significativas entre la ORS de profesionales de áreas sexualmente tipificadas. La orientación diferencial consignada en el área laboral fue parcialmente comprobada - para el caso de la masculinidad-, lo que merece entonces especial atención; pero en lo global y familiar, la femineidad y masculinidad no se estarían diferenciando entre los grupos.

En la esfera laboral, encontramos que la ORS en su aspecto de femineidad, no se relaciona con el tipo de ocupación sexualmente tipificada. Tampoco la masculinidad entendida como lo que a "masculinidad 1" concerniente a: asertivo, confía en sí mismo, independiente, seguro de sí mismo, toma decisiones con facilidad, fuerte personalidad, dominante, defiende sus creencias, capaz de tomar una posición. Sin embargo, resulta interesante notar que los atributos de "masculinidad 2", una escala independiente de la anterior, sí se relacionan con el tipo de profesión desempeñada. Estos atributos son los siguientes: activo, analítico, de empuje, con habilidades de liderazgo, deseoso de arriesgarse, agresivo, individualista y actúa como líder.

Esta segunda escala que ha sido derivada a través del modelo de Rasch, estaría apuntando a una concepción diferente de la masculinidad. En ella se agrupan los ítems que tienen una relación más directa con el control del medio y la vinculación que existe entre la persona y éste (Raguz, 1991). La primera escala de masculinidad parece poner más peso en la autopercepción, es decir, es cómo se considera el individuo a sí mismo, ya no en relación tan directa con la acción y con el medio.

Pareciera entonces que el hecho que las mujeres de profesiones tradicionalmente masculinas tengan una mayor masculinidad del segundo tipo que las de profesiones tradicionalmente femeninas podría entenderse a la luz del grupo de referencia con el que ellas trabajan. Dado que está constituido en la mayoría por hombres, las mujeres tendrían que exacerbar los rasgos de masculinidad que se expresan justamente en la interacción.

Es importante notar entonces que las mujeres que ya incursionan por esos campos tienen, a nivel psicológico, los rasgos requeridos para entrar en dicho 
mundo competitivo (la escala M2), pudiendo mantener sus roles delimitados $\mathrm{y}$ diferenciables en lo familiar y general.

La masculinidad, en la mayoría de los estudios de roles, parece ser uno de los predictores más significativos sobre distintas variables. Hemos encontrado estudios que correlacionan esta orientación con la motivación de logro (Etaugh y Petroski, 1985), expectativas de éxito (Zollo et. al. 1985), asertividad (Nix y Staufacher, 1980), autonomía para la tarea (Straub y Rodgers, 1986). Podríamos inferir entonces que las profesionales de áreas tradicionalmente masculinas en cuanto a lo laboral, estarían actualizando los rasgos ya antes mencionados.

Sin embargo, si asumimos a los roles como un conjunto de expectativas que imparte la sociedad a sus miembros acorde a su sexo biológico, estaríamos observando una relativa movilidad en la actualización de los roles profesionales; pues al no hallar correspondencia directa entre la ORS con las áreas sexualmente tipificadas podemos pensar en la probable existencia de una situación de cambio, la cual podría estar generándose tanto en la misma sociedad al no tipificar a sus miembros con la estrictez de décadas atrás. Es decir, quizás en las mujeres que trabajan y viven una experiencia de profesionalización, se daría con mayor facilidad un cuestionamiento sobre "aquellas prescripciones de conducta" (roles) con las que fueron formadas.

Asociamos dicha experiencia de profesionalización tanto a la capacidad de enjuiciamiento que se supone este proceso estimula, como a una probable "masculinización". Comprendemos esto último no en el sentido estricto de que las mujeres profesionales tengan que asumir rasgos masculinos, pero sí en el alejamiento de una femineidad tradicional, en todas las áreas. Hecho que concuerda con los resultados de Welch (1979). Vemos entonces que aunque la mayoría de las profesionales parecería no haber tenido tal influencia en su ambiente inmediato (padres), ellas habrían interiorizado modelos que les darían estabilidad en cuanto a las diferentes esferas de los roles: lo global, familiar y laboral.

Desde esta perspectiva de ser ellas portadoras de un cambio, podríamos pensar que también tuvieron un ambiente familiar facilitador para ello. $\mathrm{La}$ posición parental de conformidad con la elección vocacional de sus hijas habría ayudado al desarrollo de roles más o menos autónomos, sin una presión que tienda a la tipificación o coerción hacia modelos tradicionales, concordando con los hallazgos de Jones (1983) que los padres de las profesionales contribuyen a la meta ocupacional de sus hijas. 
Raez (1981) encuentra en su estudio con mujeres de clase media limeña que existiría un alejamiento de los modelos femeninos tradicionales. Resulta destacable la común tendencia al cambio.

Por otro lado, la consistencia trans-situacional hallada nos permite pensar en cierta estabilidad interna de las mujeres profesionales, por lo cual no coincidimos con Wojciechawski (1982), quien afirma que existe una confusión de roles al alejarse de los patrones hacia los que las mujeres fueron socializadas.

Siendo escasos los trabajos sobre roles sexuales en mujeres profesionales realizados en el Penú, a partir de nuestra experiencia nos permitimos hacer las siguientes sugerencias:

- Replicar este estudio con una variación metodologica: aplicar el SROS en sus tres versiones y el cuestionario a una misma persona lo cual permitirá hacer comparaciones más exhaustivas en términos individuales.

- Diseñar investigaciones en las cuales se homogenice mejor la muestra tomando en cuenta variables como la edad, los años de egreso de la universidad y de trabajo en su profesión.

- Proponemos también talleres de reflexión con estudiantes secundarios con miras a optimizar la orientación vocacional brindando información obtenida en las investigaciones de roles sexuales; así como programas de difusión de posibilitar el cambio de actitudes hacia éstas.

\section{Referencias}

Barrig, M. (1988). Investigación sobre el empleo y trabajo femenino: una revisión crítica. Seminario para el avance de la investigación sobre la relación de género y la situación de la mujer en la sociedad peruana. Lima: Fomciencias.

Best, D.L. (1980). An overview of findings from children's studies of sex trait stereotypes in 25 countries. Monografía presentada en la 5ta. conferencia del IACCP, India, Dic. 28, de 1980 - Enero 10.

Bohoslavsky, R. (1974). Orientación Vocacional: La Estrategia Clínica. Buenos Aires: Ediciones Nueva Vision.

Bonino, G. Kiguel, R., Santelices, R. (1980). Roles Sexuales. Construcción de un Instrumento de Medición basado en la Teoría de la Androginie Psicológica. Tesis en Psicología. Universidad Católica de Chile.

Campaña, P. (1982). Estudio Preliminar de la condición y participación económica de la mujer en el Perú Rural. Lima: Perú Mujer/AMIDEP 
Dambrot, F. \& Vassel, B. (1983). Women lawyers: the employment status of their mothers and the role models they select. Psychological Reports, 52, (1), 27-28.

Etaugh, C. \& Petrosky, B. (1985). Perceptions of women: effects on employment status and marital status. Sex Roles, 12 (3-4), 329-339.

Galejs, I. \& King, A. (1983). Sex-role perceptions of traditional and non traditional college women. Journal of Psychology, 113, (2). 257-263.

Guzman, V. (1978). Los últimos 25 años: Cambios en el trabajo femenino. En: Viva (Revista Feminista) № 9

Hawley, P. \& Even, B. (1982). Work and sex/role attitudes in relation to education and other characateristics. Vocational Guidance Quarterly, 31, (2), 101-108.

Jones, C. (1983). La dependencia de la mujer y su relación con el trabajo. Tesis de Psicología, Pontificia Universidad Católica del Perú.

Lora, C. y Anderson J. (1989). La identidad Femenina en el Contexto de la Sociedad Peruana. Dos balances sobre lo avanzado en la investigación social desde las perspectivas de la Antropología y la Psicología. Seminario sobre Género en el Perú. Organizado en colaboración con ADEC ATC; con el auspicio del Dpto de Ciencias Sociales de la Universidad Católica del Perú y del Instituto de Estudios Peruanos.

Nix, J.M., Lohr,J.M. \& Stauffacher, R. (1980). Relationship of sex.Sex Role orientation and a self-report measure of assertiveness in college students. Psychological Reports. 47 (3). 1239-1244.

Raez, M. (1981). Perfil Psicológico de la Mujer Peruana. En: Burga, T. \& M. F. Cathelat: Perfil de la Mujer Peruana, Lima, Isa-BIP.

Raguz de Romaña, M. Kerbusch, S.J. \& Van den Wolenberg, A.L. (1989). Derivation of unidimensional sample-free Sex Role Orientation Scales trough Rash-analysis. Sometido a Publicación.

Raguz de Romaña, M. (1991). Msculinity and femininity An empirical definition. Tesis doctoral. Universidad Católica de Nimega, Holanda.

Sara Lafosse, V. (1978). La Familia y la Mujer en Contextos Sociales Diferentes. Lima: Pontificia Universidad Católica del Perú.

Schaffer, D. (1979). Social and Personality Development. Monterrey: Brooks. Spence y Helmreich (1979). On assessing “androgyny”. Sex Roles, 5 (6) 721738.

Straub, C.A. \& Rodgers, R.F. (1986). An exploration of Chickering's theory and women's development. Journal of College Student Personnel. 27, (3) 216-224.

Sutherland, S.L. (1978). The unambitious female: women's low professional aspirations. Signs, 3 (4), 774-794.

Tohidi, N. (1984). Sex differences in achievement/career motivations of Iranian boys and girls. (1984). Sex Roles, 11, (5-6), 467-484.

UNICEF (1981). Participación Económica y Social de la Mujer Peruana. Lima: Feb., 1 ed. 
Welch, R.L. (1979). Androgyny and derived identity in married woman with varying degrees of non-traditional role involvement. Psychology of Women Quarterly. 3, (3), 308-315.

Williams, J.E (December 1980-January 1981). An overview of findings from adults stereotypes in young children. Monograph to the 5th Meeting IACCP India, Dec. 28th, Jan, 1980.

Williams, J.E. \& Best, D.L. (1982). Measuring sex stereotypes: Cross-cultural research and methodology. California: Sage, 1982, 6.

Zollo, L.J. Heimberg, R.G. \& Becker, R.E. (1985). Evaluations and consequences of assertive behavior. Journal of Behavior Therapy \& Experimental Psychiatry, 16, (4), 295-301. 